\title{
ALTERAÇÕES NA MICROBIOTA GASTROINTESTINAL DE CRIANÇAS COM TRANSTORNO DO ESPECTRO AUTISTA: UMA REVISÃO SISTEMÁTICA
}

DOI: 10.22289/2446-922X.V7N2A11

\author{
Fabiana do Nascimento Prazeres Martins ${ }^{1}$ \\ Leidyane Balieiro Guimarães Cunha \\ Eliza Maria da Costa Brito Lacerda
}

\section{RESUMO}

Pesquisas sobre as causas do Transtorno do Espectro Autista (TEA) têm levado vários cientistas a relacionar diversos outros sistemas ao cérebro do paciente, associando alterações na microbiota gastrointestinal (MGI) a comportamentos típicos de pessoas com TEA, sugerindo que o eixo cérebro-intestino seja uma importante peça neste 'quebra-cabeças'. Este trabalho teve por objetivo averiguar a microbiota gastrointestinal em crianças com TEA. Tal pesquisa foi realizada em plataformas de trabalhos científicos, resultando em 293 trabalhos, que, após a seleção, somaram 28 trabalhos analisados. Quase todos evidenciaram modificações na microbiota intestinal das crianças com TEA, sendo a redução da diversidade desta a principal alteração destes indivíduos em relação ao grupo controle. Destacaram-se nestes estudos a família bacteriana Lachnospiraceae, os gêneros bacterianos Lactobacillus, Clostridium, Feacalibacterium e Bacteroides; e o gênero fúngico Candida. Dentre os sintomas, a constipação foi bastante frequente nas crianças com TEA e alguns grupos de microrganismos foram associados positiva ou negativamente a alterações no sono, no desenvolvimento da fala e nos aspectos comportamentais. Concluímos que há várias alterações da MGl nas crianças com autismo quando comparado a crianças com o neurodesenvolvimento típico, sendo também afetada por questões anatômicas e metabólicas como causas ou consequências do TEA. Há necessidade de novos estudos, especialmente na população brasileira, e mais especificamente nos estados, tendo em vista a grande heterogeneidade do nosso povo e de sua cultura, as grandes variações alimentares das últimas décadas, bem como o aumento do fluxo gênico entre as populações mundiais.

Palavras-chave: Transtorno do espectro autista (TEA); Microbiota gastrointestinal (MGI); Pediatria.

CHANGES IN THE GASTROINTESTINAL MICROBIOTA OF CHILDREN WITH AUTISM SPECTRUM DISORDERS: A SYSTEMATIC REVIEW

\section{ABSTRACT}

Researchs about the causes of Autistic Spectrum Disorder (ASD) has led several scientists to relate many others systems to the patient's brain, linking changes in the gastrointestinal microbiota (GIM) to typical behaviors of people with ASD, suggesting that the brain-intestine axis is an important piece in this 'puzzle'. This work aimed to investigate the microbiota

\footnotetext{
${ }^{1}$ Endereço eletrônico de contato: fnp.biologa@gmail.com

Recebido em 17/06/2021. Aprovado pelo conselho editorial para publicação em 24/09/2021.
} 
gastrointestinal in children with ASD. Such research was carried out on scientific work platforms, resulting in 293 works, which, after the selection, totaled 28 works analyzed. Almost all of them showed changes in the intestinal microbiota of children with ASD, the reduction of their diversity being the main alteration of these individuals in relation to the control group. The bacterial family Lachnospiraceae, the bacterial gender Lactobacillus, Clostridium, Feacalibacterium, and Bacteroides stood out in these studies; and the fungal genus Candida. Among the symptoms, constipation was quite frequent in children with ASD and some groups of microorganisms were positively or negatively associated with changes in sleep, speech development and behavioral aspects. We conclude that there are several alterations of GIM in children with autism when compared to children with typical neurodevelopment, being also affected by anatomical and metabolic issues as causes or consequences of ASD. There is a need for further studies, especially in the Brazilian population, and more specifically in the states, in view of the great heterogeneity of our people and their culture, the great dietary variations of the last decades, as well as the increase in gene flow among world populations.

Keywords: Autism Spectrum Disorders (ASD); gastro-intestinal microbiota (GIM); Pedriatrics.

\section{ALTERACIONES EM LA MICROBIOTA INTESTINAL DE NIÑOS CON TRANSTORNO DEL ESPECTRO AUTISTA: UNA REVISIÓN SISTEMÁTICA}

\section{RESUMEN}

Investigaciones sobre las causas del TRANSTORNO DEL ESPECTRO AUTISTA (TEA) ha llevado a varios científicos a relacionar otros sistemas del cerebro del paciente, asociando alteraciones en el microbiota gastrointestinal (MGI) a comportamientos típicos de personas con TEA, sugiriendo que el eje cerebro-intestino sea una importante pieza en este rompe cabeza. Este trabajo tiene por objetivo averiguar a microbiota gastrointestinal en niños con TEA. Tal investigación fue realizada en plataformas de trabajos científicos, resultando en 293 que después de la selección, sumaron 28 trabajos analizados. Casi todos evidenciaron modificaciones en la microbiota intestinal de los niños con TEA, siendo la reducción la diversidad de esta la principal alteración de estos individuos en relación al grupo control. Destacándose en estos estudios la familia bacteriana Lactobacillus, Clostridium, Feacalibacterium, y bacteroides, y el género Fúngico Candida. Entre los síntomas, la constipación fue bastante frecuente en los niños con TEA y algunos grupos de microorganismos fueron asociados positiva o negativamente a alteraciones de sueño, en el desenvolvimiento del lenguaje y en los aspectos de comportamiento. Concluimos que hay varias alteraciones de MGI en los niños con autismo cuando lo comparamos en niños con el neurodesenvolvimiento típico, siendo también afectada por cuestiones anatómicas y metabólicas como causas o consecuencias del TEA. Hay necesidad de nuevos estudios, especialmente en la populación peruana, y más específicamente en provincias teniendo en cuenta la grande heterogeneidad de nuestro pueblo y de su cultura, las grandes variaciones alimenticias de las últimas décadas, como el aumento de flujo genético entre las poblaciones mundiales.

Palabras claves: Trastorno del Espectro Autista (TEA); Microbiota gastrointestinal (MGI); Pediatría. 


\section{INTRODUÇÃO}

O transtorno do espectro autista (TEA), amplamente conhecido como autismo, é um transtorno do desenvolvimento cujas características envolvem o comprometimento qualitativo da interação social recíproca, da comunicação, e um repertório restrito de atividades e interesses que devem estar presentes antes dos três anos de idade American Psychiatric Association [APA] (2002).

"O diagnóstico do autismo infantil é baseado principalmente no quadro clínico do paciente, não havendo ainda um biomarcador que o caracterize" Da Costa, \& Nunesmaia (1998). "Entretanto, existem fatores genéticos como deleções no gene da neurexina 1, duplicações em 7q11.23 e 15q11-13 e duplicações ou deleções em 16p11.2, além de marcadores metabólicos e mitocondriais não exclusivos para o TEA" (Ratajczak, 2011).

"Existem vários princípios utilizados para a classificação do autismo, sendo os principais o Manual de Diagnóstico e de Estatística de Doenças Mentais da Academia Americana de Psiquiatria, DSM, e a Classificação Internacional de Doenças da Organização Mundial da Saúde" Mello (2001). "As causas do TEA são diversas, quase sempre multifatoriais, envolvendo fatores genéticos e ambientais, sendo associado constantemente a doenças autoimunes e outras disfunções do sistema imunológico, além da predisposição às disfunções gastrointestinais de grande prevalência" (Hughes, Rose, \& Ashwood, 2018).

A disfunção da microbiota ou disbiose é caracterizada por alterações na proporção de Firmicutes/ Bacteroides, divisões bacterianas dominantes no intestino, e na composição de filos bacterianos primários (Firmicutes, Bacteroidetes, Fusobacteria e Verrucomicrobia). Várias interações do eixo microbiota-intestino-cérebro são multifatoriais e não completamente definidas. O sistema nervoso entérico atua como um condutor de comunicação entre a microbiota gastroinstestinal (MGI) e o sistema nervoso central (SNC) (De Angelis, Francavilla, Piccolo, De Giacomo \& Gobbetti, 2015).

Nos últimos anos, a MGI tem se destacado com grande potencial para afetar funções cognitivas, influenciar distúrbios do neurodesenvolvimento, ansiedade, depressão, alterações comportamentais, e outros sintomas associados ao TEA. Alterações na MGI em crianças com TEA têm sido reportadas e associações entre o gênero microbial e os sintomas desse transtorno têm sido descritas (Tomova et al., 2015).

A MGI influencia o cérebro e pode estar envolvida em distúrbios neuropsiquiátricos, em parte por modular a disponibilidade de triptofano circulante, serotonina, quinurenina e ácidos graxos de cadeia curta. Está ligada também à permeabilidade da barreira hematoencefálica e à ativação de células imunológicas periféricas e células da Glia. O eixo intestino-cérebro envolve sinalização bioquímica entre o trato gastrointestinal e o SNC. Quando a estrutura gastrointestinal está comprometida, a funcionalidade protetora da barreira fica deficiente, gerando um aumento na permeabilidade e, consequentemente, a penetração de substâncias Rev. Psicol Saúde e Debate. Set., 2021:7(1): 169-180 
que podem alterar a função fisiológica (Turner, 2009 como citado em Generoso, Giridharan, Macedo, \& Barichello, 2020).

O objetivo desse estudo foi descrever as possíveis relações e influências da MGI na manifestação de sintomas do TEA em crianças.

\section{DESENVOLVIMENTO}

Esta pesquisa se caracterizou pelo levantamento bibliográfico sobre pesquisas que relacionem sintomas apresentados por crianças no transtorno do espectro autista a alterações da MGI.

O levantamento de literatura foi realizado em plataformas de buscas referências sobre artigos científicos na área da saúde: SCIELO, PUBMED, LILACS e MEDLINE. O período de acesso foi nos meses de janeiro, fevereiro e março de 2021. As palavras chaves utilizadas foram: transtorno do espectro autista; microbiota gastrointestinal; e crianças; e suas respectivas traduções para a língua inglesa: autism spectrum disorders; gastrointestinal microbiota; e children.

Como critérios de inclusão foram utilizados artigos apresentando as palavras-chave citadas e que tratavam do tema TEA na infância e microbiota gastrointestinal, relacionando-os entre si.

Os critérios de exclusão consideraram artigos que não abordavam o tema na íntegra, ou tinham um caráter superespecializados, por exemplo, utilizando como base o uso de probióticos, ou relacionando as alterações na microbiota gastrointestinal a uso de determinadas substâncias.

Esta pesquisa encontrou 293 publicações nas quatro plataformas utilizadas para a realização da busca. Após a seleção usando os critérios de exclusão já citados, restaram 28 trabalhos para análise. Os estudos avaliaram as alterações pela diversidade e estrutura da MGI, através de metodologias de identificação dos microrganismos e também da quantificaçã o de seus metabólitos.

Entre trabalhos analisados, todos evidenciaram alguma alteração na microbiota gastrointestinal em crianças com TEA em relação ao grupo controle. As avaliações da diversidade e abundância dos artigos foram a níveis de família, gênero ou espécie. Dois trabalhos (Ma et al., 2019; Son et al., 2015) não observaram alterações significativas nos filos, identificaram a nível de gênero diferenças com relação ao grupo controle.

A imensa maioria dos trabalhos analisados avaliou apenas a microbiota bacteriana, sendo que apenas um tratava sobre alterações na composição fúngica da microbiota Zou et al. (2021); e somente um dos artigos avaliou a presença de bactérias e fungos concomitantes na microbiota individual (Moreno et al., 2015; Strati et al., 2017). 
A alteração mais descrita foi a diminuição da diversidade na microbiota intestinal (Averina et al., 2020; Carissimi et al., 2019; Ma et al., 2019), embora alguns autores tenham relatado o aumento desta diversidade em indivíduos com TEA (De Angelis et al., 2013; Tomova et al., 2020).

Alguns autores relataram diminuição na abundância relativa (Liu et al., 2019; Ma et al., 2019) e alterações na composição da família Lachnospiraceae (De Angelis et al., 2013), enquanto outros encontraram aumento da abundância relativa desta mesma família de bactérias (Ding et al., 2020).

Os Lactobacillus, em geral, estão em quantidade elevadas em crianças com TEA (Strati et al., 2017; Tomova et al., 2015), mas também alguns autores não consideraram alterações significativas no nível deste gênero bacteriano (Sun, You, Jia, \& Wang, 2019).

A família Clostridiaceae esteve presente em um nível mais alto Sun, et al. (2019), estando significativamente aumentada no nível da ordem Clostridiales Ding et al. (2020), sendo a Clostridium ssp encontrada em maior quantidade no TEA (De Angelis et al., 2013; Strati et al., 2017). Houve também diferenças na desta família bacteriana em crianças com TEA em relação ao grupo controle (Zhang, Ma, Zhang, He, \& Wang, 2018).

Em relação ao filo Bacteriodetes, a maioria dos trabalhos relatavam sua diminuição (Ding et al., 2020; Strati et al., 2017; Tomova et al., 2015), porém alguns autores descreveram aumento neste filo entre as crianças com TEA (Coretti et al., 2018; De Angelis et al., 2013).

O gênero Feacalibacterium estava diminuído na maioria dos estudos em crianças com autismo (Ding et al., 2020; Kang et al., 2018; Sun et al., 2019), mas houve evidência de aumento nesse gênero em alguns estudos Inoue et al. (2016). Em outros trabalhos, Faecalibacterium prausnitzii estava em nível elevado e foi considerado um colonizador tardio do intestino saudável (Coretti et al., 2018).

Quanto à microbiota fúngica, o gênero Candida apareceu em quantidade dobrada em crianças com TEA (Strati et al., 2017), sendo um dos principais agentes causadores da disbiose (Moreno et al., 2015). Também foram observadas alterações na microbiota fúngica relacionadas a Aspergillus ssp. e Saccharomyces cerevisiae, sendo este aumentado e aquele observado em menor abundância (Zou et al., 2021).

Houve relação positiva entre o grau de comprometimento do TEA e a gravidade da disfunção da microbiota (Ding et al., 2020; Kang et al., 2013; Tomova et al., 2015), sendo que o principal sintoma relatado entre as crianças com TEA foi a constipação (Dan et al., 2020; Kushak et al., 2017; Liu et al., 2019;). Enterococcus foi positivamente associado à regressão mental em crianças com TEA (Plaza-Diaz et al., 2019). Para Hua 2020, os níveis elevados de Faecalibacterium e Agathobacter pareceram estar associados a distúrbios do sono. Outras mudanças em alguns casos não se mostraram significativamente importantes para a sintomatologia do TEA (Ahmed et al., 2020). 
Alguns autores associaram diretamente as alterações encontradas a distúrbios no sistema imune (Inoue et al., 2016; Zhang et al., 2018; Zou et al., 2021), e, mesmo que de forma indireta, a relação entre o sistema imune e a microbiota foi evidenciada na maioria dos trabalhos.

Os mecanismos envolvidos na relação microrganismo e sintomatologia do TEA são diversos, podendo estar relacionados à própria função da bactéria ou do fungo; às vezes o metabólito liberado pelo microrganismo é que provoca o sintoma (Kang et al., 2018; Kang et al., 2020). Houve também evidência na relação da microbiota intestinal com a expressão gênica (Inoue et al., 2016).

Esta revisão de literatura demonstrou que há evidências que alterações na microbiota intestinal possam estar relacionadas à sintomatologia apresentada pelas crianças com transtorno do espectro autista. No entanto, estas alterações não possuem um padrão, podendo ser bem diversificadas (Ho et al., 2020).

A regionalidade parece ser um fator a ser observado, pois a dieta e os hábitos de higiene são fortemente influenciados pela cultura, e esses por sua vez interferem na estrutura da microbiota gastrointestinal dos indivíduos (Zhang et al., 2018).

Cada micro-organismo tem o seu grau de contribuição seja ele benéfico ou maléfico, mas no caso da microbiota gastrointestinal, a estrutura tem uma grande importância porque a forma de organização e distribuição confere ou não o equilíbrio necessário ao seu bom funcionamento do intestino (Cryan et al., 2019).

De acordo com este levantamento, um dos principais problemas na microbiota das crianças com TEA, foi o desequilíbrio. A maioria dos estudos apontou baixa diversidade, isso significa ter muito de certo grupo específico ou de uma espécie de microrganismo, e ter pouco ou não ter representantes de outros grupos de bactérias, fungos ou vírus (Carissimi et al., 2019).

As alterações na MGI podem estar associada à seletividade alimentar, sintoma muito comum no TEA, tornando-se um ciclo vicioso, onde a microbiota alterada desencadeia ou acentua sintomas do TEA, e tais sintomas promovem alterações indesejáveis na microbiota (Cupertino et al., 2018; Gondolia et al., 2012).

Estudos recentes têm elucidado como a microbiota pode influenciar outros sistemas do organismo. Desde a alteração na impermeabilidade do intestino, até a liberação de substâncias que podem ser tóxicas e interferir na expressão gênica, além de uma interferência direta no sistema imune (Averina et al., 2020; Kang et al., 2018).

As manifestações são diferentes em cada indivíduo, o que demanda maiores estudos envolvendo diferentes áreas médicas e científicas para a investigação desse distúrbio. Como a composição da microbiota também é afetada por questões anatômicas e metabólicas, não é possível padronizar uma microbiota ideal para populações ou grupo de indivíduos. Assim, 
todas as investigações diagnósticas e eventuais tratamentos precisam ser completamente individualizados (De Angelis, 2013; Son et al., 2015)

\section{CONSIDERAÇÕES FINAIS}

Em resumo, este trabalho demonstra ser importante um olhar integrativo sobre o paciente com Transtorno do Espectro Autista, sendo indispensável a observação da saúde gástrica intestinal, e outros sistemas, como o endócrino e o sistema imune, pois todos eles se relacionam. Como o intestino é a maior porta de entrada das substâncias no corpo, alterações no seu funcionamento podem ser repercutidas qualquer órgão ou sistema, mas o cérebro é especialmente sensível a estes distúrbios.

Fica evidente também a necessidade de novos estudos sobre a importância na microbiota para o desenvolvimento das crianças com TEA, especialmente na população brasileira, tendo em vista a grande heterogeneidade do nosso povo e de sua cultura, as grandes variações alimentares das últimas décadas, bem como o aumento do fluxo gênico entre as populações mundiais.

Conhecer o perfil da microbiota das nossas crianças e apontar suas alterações pode ser um dos caminhos para oferecer tratamento seguro e individualizado, melhor resposta às condutas médicas, maior qualidade de vida, e ao que tudo indica, a oportunidade de um melhor desenvolvimento.

\section{REFERÊNCIAS}

Ahmed, S.A.S., Elhefnawy, A. M., Azouz, H. G., Roshdy, Y. S., Ashry, M. H., Ibrahim, A. E., Meheissen, M. A. (2020). Study of the gut Microbiome Profile in Children with Autism Spectrum Disorder: a Single Tertiary Hospital Experience. J Mol Neurosci. Jun;70(6):887-896. Disponível em: https://pubmed.ncbi.nlm. nih.gov/ 32062762/ Acesso em: 13/01/2021.

American Psychiatric Association (2002). DSM-IV-TR: Manual diagnóstico e estatístico de transtornos mentais (C. Dornelles, Trad., 4. ed. rev.). Porto Alegre, RS: ArtMed.

Averina, O.V., Kovtun, A.S., Polyakova, S.I., Savilova, A.M., Rebrikov, D.V., Danilenko, V.N. (2020). The bacterial neurometabolic signature of the gut microbiota of young children with autism spectrum disorders. J Med Microbiol Apr;69(4):558-571. Disponível em: https://pubmed.ncbi.nlm.nih. gov/32213246/

Carissimi, C., Laudadio, I., Palone, F., Fulci, V., Cesi, V., Cardona, F., Alfonsi, C., Cucchiara, S., Isoldi, S., Stronati, L. (2019). Functional analysis of gut microbiota and immunoinflammation in children with autism spectrum disorders. Dig Liver Dis. Oct;51(10):1366-1374. Disponível em: https://pubmed.ncbi.nlm. nih.gov/ 31320306/

Coretti, L., Paparo, L., Riccio, M. P., Amato, F., Cuomo, M., Natale, A., Borrelli, L., Corrado, G., Comegna, M., Buommino, E., Castaldo, G., Bravaccio, C., Chiariotti, L., Canani, R. B., Lembo, F. (2018). Gut Microbiota Features in Young Children With Autism Spectrum Disorders. Front Microbiol Dec 19;9:3146. Disponível em: https://pubmed.ncbi.nlm.nih.gov/30619212/ 
Costa, M.I.F., \& Maia, H.G.S. (1998). Diagnóstico genético e clínico do autismo infantil. Arq. Neuro-Psiquiatr. vol.56 n.1 São Paulo, Mar. Disponível em: https://www.scielo.br/scielo.php?pid=S0004282X1998000100004\&script=sci_abstrac t\&tlng=PT

Cryan, J., O'Riordan, K. J., Cowan, C. S. M., Sandhu, K. V., Bastiaanssen, T. F. S., Boehme, M., Codagnone, M. G., Cussotto, S., Fulling, C., Golubeva, A. V., Guzzetta, K. E., Jaggar, M., Long-Smith, C. M., Lyte, J. M., Martin, J. A., Molinero-Perez, A., Moloney, G., Morelli, E., Morillas, E., O'Connor, R., Cruz-Pereira, J. S., Peterson, V. L., Rea, K., Ritz, N. L., Sherwin, E., Spichak, S., Teichman, E. M., Van De Wouw, M., Ventura-Silva, A. P., Wallace-Fitzsimons, W. E., Hyland, N., Clarke, G., Dinan, T. G. (2019). The Microbiota-Gut-Brain Axis. Physiol Rev. Oct 1;99(4):1877-2013. Disponível em: https://journals.physiology.org/doi/pdf/10.1152/ physrev. 00018.2018

Cupertino, M.C., Resende, M. B., Veloso, I. F., Carvalho, C. A., Duarte, V. F., Ramos, G. A. (2019). Transtorno do espectro autista: uma revisão sistemática sobre aspectos nutricionais e eixo intestino-cérebro/ Autism spectrum disorder: a systematic review about nutritional aspects and gut-brain axis. ABCS health sci ; 44(2): 120-130, 11 out. Disponível em: https://docs.bvsalud.org/biblioref/2019/ 10/1022353/44abcs120.pdf

Dan, Z., Mao, X., Liu, Q., Guo, M., Zhuang, Y., Liu, Z., Chen, K., Chen, J., Xu, R., Tang, J., Qin, L., Gu, B., Liu, K., Su, C., Zhang, F., Xia, Y., Hu, Z., Liu, X. (2020). Altered gut microbial profile is associated with abnormal metabolism activity of Autism Spectrum Disorder. Gut Microbes Sep 2;11(5):1246-1267. Disponível em: https://pubmed.ncbi.nlm.nih.gov/32912596/

De Angelis, M., Piccolo, M., Vannini, L., Siragusa, S., DE Giacomo, A., Serrazzanetti, D.I., Cristofori, F., Guerzoni, M.E., Gobbetti, M., Francavilla, R. (2013). Fecal microbiota and metabolome of children with autism and pervasive developmental disorder not otherwise specified. PLoS One Oct; 9;8(10):e76993. Disponível em: https://pubmed.ncbi.nlm.nih.gov/ 24130822/

De Angelis, M; Francavilla, R; Piccolo, M; De Giacomo, A; Gobbetti, M. (2015). Autism spectrum disorders and intestinal microbiota. Gut Microbes 6(3):207-13. Disponível em: https://pubmed.ncbi.nlm.nih.gov/ 25835343/

Ding, X., Xu, Y., Zhang, X., Zhang, L., Duan, G., Song, C., Li, Z., Yang, Y., Wang, Y., Wang, X., Zhu, C. (2020). Gut microbiota changes in patients with autism spectrum disorders. J Psychiatr Res. Oct;129:149-159. Disponível em: https://pubmed.ncbi.nlm.nih.gov/32912596/

Generoso, J.S., Giridharan, V. V., Lee, J., Macedo, D., Barichello, T. (2020). The role of the microbiota-gut-brain axis in neuropsychiatric disorders. Braz. J. Psychiatry, ahead of print Epub July 10. Print version ISSN 1516-4446 On-line version ISSN 1809-452X. Disponível em: https://www.scielo.br/scielo. php?script=sci_arttext\&pid=S151644462020005021202.

Gondalia, S.V., Palombo, E. A., Knowles, S. R., Cox, S.B., Meyer, D., Austin, D.W. (2012). Molecular characterization of gastrointestinal microbiota of children with autism (with and without gastrointestinal dysfunction) and their neurotypical siblings. Autism Res. Dec;5(6):419-27. Disponível em: https://pubmed.ncbi.nlm.nih.gov/22997101/

Ho, L. K. H., Tong, V. J. W., Syn, N., Nagarajan, N., Tham, E. H., Tay, S. K., Shorey, S., Tambyah, P. A., Law, E. C. N. (2020). Gut microbiota changes in children with autism spectrum disorder: a systematic review. Gut Pathog. Feb 3;12:6.doi: 10.1186/s13099020-0346-1. eCollection. Disponível em: https://www.ncbi. nlm.nih.gov/pmc/ articles/PMC6996179/

Hua, X., Zhu, J., Yang, T., Guo, M., LI, Q., Chen, J., LI, T. (2020). The Gut Microbiota and Associated Metabolites Are Altered in Sleep Disorder of Children With Autism Spectrum Disorders. Front Psychiatry Sep 2;11:855. Disponível em: https://pubmed.ncbi.nlm.nih.gov/32982808/ Acesso em: 20/01/2021

Hughes, H.K., Rose, D., Ashwood, P. (2018). The Gut Microbiota and Dysbiosis in Autism Spectrum Disorders. Curr Neurol Neurosci Rep. 18(11): 81. Disponível em: https://pubmed.ncbi.nlm.nih.gov/30251184/ 
Inoue, R., Sakaue, Y., Sawai, C., Sawai, T., Ozeki, M., Romero-Pérez, G. A., Tsukahara, T. (2016). A preliminary investigation on the relationship between gut microbiota and gene expressions in peripheral mononuclear cells of infants with autism spectrum disorders.
Biosci
Biotechnol
Biochem. Dec;80(12):2450-2458.
Disponível em: https://pubmed.ncbi.nlm.nih.gov/27581276/

Kang, D. W., Ihan, Z.E., Isern, N.G., Hoyt, D.W., Howsmon, D.P., Shaffer, M., Lozupone, C.A., Hahn, J., Adams, J.B., Krajmalnik-Brown, R. (2018). Differences in fecal microbial metabolites and microbiota of children with autism spectrum disorders. Anaerobe Feb; 49:121-131. Disponível em: https://pubmed.ncbi.nlm.nih.gov/29274915/

Kang, D. W., Adams, J. B., Vargason, T., Santiago, M., Hahn, J., Krajmalnik-Brown, R. (2020). Autism Spectrum Disorders and Their Modulation after Microbiota Transfer Therapy. mSphere Oct 21;5(5):e00314-20. Disponível em: https://pubmed.ncbi.nlm. nih.gov/ 33087514/ Acesso em: 03/02/2021.

Kang, D. W., Park, J. G., Ilhan, Z. E., Wallstrom, G., Labaer, J., Adams, J. B., KrajmalnikBrown, R. (2013). Reduced incidence of Prevotella and other fermenters in intestinal microflora of autistic children. PLoS One Jul 3;8(7):e68322. Disponível em: https://pubmed.ncbi.nlm.nih.gov/23844187/

Kushak, R. I., Winter, H. S., Buie, T. M., Cox S. B., Phillips C. D., Ward N. L. (2017). Analysis of the Duodenal Microbiome in Autistic Individuals: Association With Carbohydrate Digestion. J Pediatr Gastroenterol Nutr; 64(5):e110-e116, May 15364801. Disponível em: https://pubmed.ncbi.nlm. nih.gov/27811623/

Laue, H. E., Korrick, S. A., Baker, E. R., Karagas, M. R., Madan, J. C. (2020). Prospective associations of the infant gut microbiome and microbial function with social behaviors related to autism at age 3 years. Sci Rep. Sep 23;10(1):15515. Disponível em: https://www.nature.com/articles/ s41598-020-72386-9.

Liu, S., Li, E., Sun, Z., Fu, D., Duan, G., Jiang, M., Yu, Y., Mei, L., Yang, P., Tang, Y., Zheng, P. (2019). Altered gut microbiota and short chain fatty acids in Chinese children with autism spectrum disorder. Sci Rep Jan; 22;9(1):287. Disponível em: https://pubmed.ncbi.nlm.nih.gov/30670726/

Ma, B., Liang, J., Dai, M., Wang, J., Luo, J., Zhang, Z., Jing, J. (2019). Altered Gut Microbiota in Chinese Children With Autism Spectrum Disorders. Front Cell Infect Microbiol Mar; 6;9:40. Disponível em: https://pubmed.ncbi.nlm. nih.gov/30895172/

Mello, A. M. S. R. Autismo: guia prático. 2ª ed. São Paulo, Corde, 2001.

Moreno, X., Santamaria, G., Sánchez, R., De La Torre, B., Garcés, F., Hernández, C., Martínez, C., Márquez, A., López, K. (2015). Microbiota gastrointestinal aeróbica en niños con trastorno del espectro autista. Estudio preliminar / gastrointestinal microbiota aerobic in children with autism spectrum disorders. Preliminary study. GEN ; 69(2): 3644, jul. Disponível em: http://bases.bireme.br/cgibin/wxislind.exe/iah/online/? IsisScript=iah/iah.xis\&src=google \&base=LILACS\&lang=p\&nextAction=Ink\&exprSearch=780150\&indexSearch=ID.

Plaza-Díaz, J., Gómez-Fernández, A., Chueca, N., De La Torre-Aguilar, M. J., Gil, A., Perez-Navero, J. L., Flores-Rojas, K., Martín-Borreguero, P., Solis-Urra, P., RuizOjeda, F. J., Garcia, F., Gil-Campos, M.(2019). Autism Spectrum Disorder (ASD) with and without Mental Regression is Associated with Changes in the Fecal Microbiota. Nutrients Feb 5;11(2):337. Disponível em: https://pubmed.ncbi.nlm.nih.gov/ 30764497/

Ratajczak, H. V. (2011). Theoretical aspects of autism: biomarkers: a review. J Immunotoxicol Jan-Mar; 8(1):80-94. Disponível em: https://pubmed.ncbi.nlm .nih.gov/21299356/

Son, J. S., Zheng, L. J., Rowehl, L. M., Tian, X., Zhang, Y., Zhu, W., Litcher-Kelly, L., Gadow, K. D., Gathungu, G., Robertson, C. E., IR, D., Frank, D. N., LI, E. (2015). Comparison of Fecal Microbiota in Children with Autism Spectrum Disorders and Neurotypical Siblings in the Simons Simplex Collection. PLoS One Oct 1;10(10):e0137725. Disponível em: https://pubmed.ncbi. nlm.nih.gov/26427004/

Strati, F., Cavalieri, D., Albanese, D., De Felice, C., Donati, C., Hayek, J., Jousson, O., Leoncinl, S., Renzi, D., Calabrò, A., De Filippo, C. (2017). New evidences on the 
altered gut microbiota in autism spectrum disorders. Microbiome Feb 22;5(1):24. Disponível em: https://pubmed.ncbi. nlm.nih.gov/28222761/.

Sun, H; You, Z; Jia, L; Wang, F. (2019). Autism spectrum disorder is associated with gut microbiota disorder in children. BMC Pediatr Dec; 27;19(1):516. Disponível em: https://bmcpediatr.biomedcentral.com/articles/10.1186/s12887-019-1896-6.

Tomova, A., Husarova, V., Lakatosova, S., Bakos, J., Vlkova, B., Babinska, K., Ostatnikova, D. (2015). Gastrointestinal microbiota in children with autism in Slovakia. Physiol Behav Jan; 138:179-87. Disponível em: https://pubmed.ncbi.nlm. nih.gov/25446201/.

Tomova, A., Soltys, K., Repiska, G., Palkova, L., Filcikova, D., Minarik, G., Turna, J., Prochotska, K., Babinska, K., Ostatnikova, D. (2020). Specificity of gut microbiota in children with autism spectrum disorder in slovakia and its correlation with astrocytes activity marker and specific behavioural patterns. Physiol Behav. Feb 1;214:112745. Disponível em: https://pubmed.ncbi.nlm. nih.gov/31765662/.

Zhai, Q., Cen, S., Jiang, J., Zhao, J., Zhang, H., Chen, W. (2019). Disturbance of trace element and gut microbiota profiles as indicators of autism spectrum disorder: A pilot study of Chinese children. Environ Res. Apr; 171:501-509. Disponível em: https://pubmed.ncbi.nlm.nih.gov/ 30743242/

Zhang, M., Ma, W., Zhang, J., He, Y., Wang, J.(2018). Analysis of gut microbiota profiles and microbe-disease associations in children with autism spectrum disorders in China. Sci Rep Sep 18;8(1):13981. Disponível em: https://www.nature.com/articles/ s41598018-32219-2.

Zhao, R.H., Zheng, P.Y., Liu, S. M., Tang, Y. C., Li, E. Y., Sun, Z. Y., Jiang, M. M. (2019). Correlation between gut microbiota and behavior symptoms in children with autism spectrum disorder. Zhongguo Dang Dai Er Ke Za Zhi. Jul;21(7):663-669. Disponível em: https://pubmed.ncbi .nlm.nih.gov/ 31315765/

Zou, R., Wang, Y., Duan, M., Guo, M., Zhang, Q., Zheng, H. (2021). Dysbiosis of Gut Fungal Microbiota in Children with Autism Spectrum Disorders. J Autism Dev Disord. Jan;51(1):267-275. Disponível em: https://pubmed.ncbi.nlm. nih.gov/32447559/

\section{ANEXOS}

\begin{tabular}{|l|l|}
\hline \multicolumn{1}{|c|}{ PRINCIPAIS ACHADOS EM CRIANÇAS COM TEA } & \multicolumn{1}{c|}{$\begin{array}{c}\text { AUTOR/ ANO DE } \\
\text { PUBLICAÇÃO }\end{array}$} \\
\hline Menor diversidade na microbiota intestinal. & $\begin{array}{l}\text { Ma et al., 2019; } \\
\text { Averina et al., 2020; } \\
\text { Carissimi et al., 2019. }\end{array}$ \\
\hline Maior diversidade na microbiota intestinal. & $\begin{array}{l}\text { De Angelis et al., 2013; } \\
\text { Tomova et al., 2020. }\end{array}$ \\
\hline Diminuição na abundância relativa da família Lachnospiraceae. & $\begin{array}{l}\text { Ma et al., 2019; } \\
\text { Liu et al., 2019. }\end{array}$ \\
\hline Alterações na composição da família Lachnospiraceae. & De Angelis et al., 2013. \\
\hline Aumento da abundância relativa de Lachnospiraceae. & Ding et al., 2020. \\
\hline Aumento da abundância relativa de Lactobacillus. & $\begin{array}{l}\text { Tomova et al., 2015; } \\
\text { Strati et al., 2017. }\end{array}$ \\
\hline Aumento da abundância de Clostridiaceae. & Sun et al., 2019. \\
\hline
\end{tabular}

Rev. Psicol Saúde e Debate. Set., 2021:7(1): 169-180 


\begin{tabular}{|c|c|}
\hline Diferença na diversidade do grupo Clostridiaceae & Zhang et al., 2018. \\
\hline Aumento significativo do gênero Clostridiales. & Ding et al., 2020. \\
\hline Maior quantidade da espécie Clostridium ssp. & $\begin{array}{l}\text { De Angelis et al., 2013; } \\
\text { Strati et al., } 2017 .\end{array}$ \\
\hline Diminuição da abundância relativa do filo Bacteriodetes. & $\begin{array}{l}\text { Tomova et al., 2015; } \\
\text { Strati et al., 2017; } \\
\text { Ding et al., 2020. }\end{array}$ \\
\hline Aumento da abundância relativa do filo Bacteriodetes & $\begin{array}{l}\text { De Angelis et al., 2013; } \\
\text { Coretti et al., 2018. }\end{array}$ \\
\hline Diminuição da abundância relativa do gênero Feacalibacterium & $\begin{array}{l}\text { Kang et al., 2018; } \\
\text { Sun et al., 2019; } \\
\text { Ding et al., 2020. }\end{array}$ \\
\hline Aumento da abundância relativa do gênero Feacalibacterium & Inoue et al., 2016. \\
\hline Espécie Faecalibacterium prausnitzii em nível elevado. & Coretti et al., 2018. \\
\hline Aumento em dobro do gênero Candida & Strati et al., 2017. \\
\hline Gênero Candida como um dos principais responsáveis pela disbiose & Moreno et al., 2015. \\
\hline Aumento da espécie Aspergillus ssp. na microbiota fúngica. & Zou et al., 2021. \\
\hline $\begin{array}{l}\text { Diminuição da abundância relativa da espécie Saccharomyces } \\
\text { cerevisisae na microbiota fúngica. }\end{array}$ & Zou et al., 2021. \\
\hline $\begin{array}{l}\text { Relação positiva entre grau de comprometimento do TEA e gravidade } \\
\text { da disfunção da microbiota. }\end{array}$ & $\begin{array}{l}\text { Tomova et al., 2015; } \\
\text { Ding et al., 2020; } \\
\text { Kang et al., 2013. }\end{array}$ \\
\hline Constipação como principal sintoma em crianças com TEA. & $\begin{array}{l}\text { Liu et al., 2019; } \\
\text { Dan et al., 2020; } \\
\text { Kushak et al., } 2017 .\end{array}$ \\
\hline $\begin{array}{l}\text { Alterações encontradas diretamente associadas aos distúrbios no } \\
\text { sistema imune. }\end{array}$ & $\begin{array}{l}\text { Zhang et al., 2018; } \\
\text { Zou et al., 2021; } \\
\text { Inoue et al., 2016. }\end{array}$ \\
\hline $\begin{array}{l}\text { Metabólito liberado pelo micro-organismo provoca sintomatologia do } \\
\text { TEA. }\end{array}$ & $\begin{array}{l}\text { Kang et al., 2018; } \\
\text { De Angelis et al., 2015; } \\
\text { Kang et al., 2020. }\end{array}$ \\
\hline $\begin{array}{l}\text { Evidência na relação entre a microbiota intestinal com a expressão } \\
\text { gênica. }\end{array}$ & Inoue et al., 2016. \\
\hline $\begin{array}{l}\text { Sugere que as alterações na microbiota de crianças com TEA estejam } \\
\text { relacionados à ansiedade e à dieta restrita. }\end{array}$ & Gondolia et al., 2012. \\
\hline $\begin{array}{l}\text { O nível de Bifidobacterium estava bem diminuído em crianças com TEA } \\
\text { quando comparado aos seus irmãos com neurodesenvolvimento típico. }\end{array}$ & Ahmed et al., 2020. \\
\hline $\begin{array}{l}\text { Faecalibacterium and Agathobacter estavam com níveis elevados em } \\
\text { crianças com TEA e distúrbios do sono. }\end{array}$ & Hua et al., 2020 \\
\hline
\end{tabular}

\begin{tabular}{|l|l|l|}
\hline Microrganismo & Sintoma associado & Presença no TEA \\
\hline
\end{tabular}




\begin{tabular}{|l|l|l|}
\hline Clostridium cluster & Constipação & Aumentada \\
\hline Escherichia & Constipação & Aumentada \\
\hline Shigella & Constipação & Aumentada \\
\hline Megamonas & $\begin{array}{l}\text { Melhor saúde física, comportamento, linguagem e } \\
\text { comunicação. }\end{array}$ & Relativa \\
\hline $\begin{array}{l}\text { Proteobacteria e o } \\
\text { filo Actinobacteria }\end{array}$ & Regressão mental & Relativa \\
\hline Agathobacter & Melhora da linguagem e do sono. & Relativa \\
\hline Faecalibacterium & Melhora o sono & Diminuída \\
\hline Blautia producta & Piora o comportamento & \\
\hline
\end{tabular}

